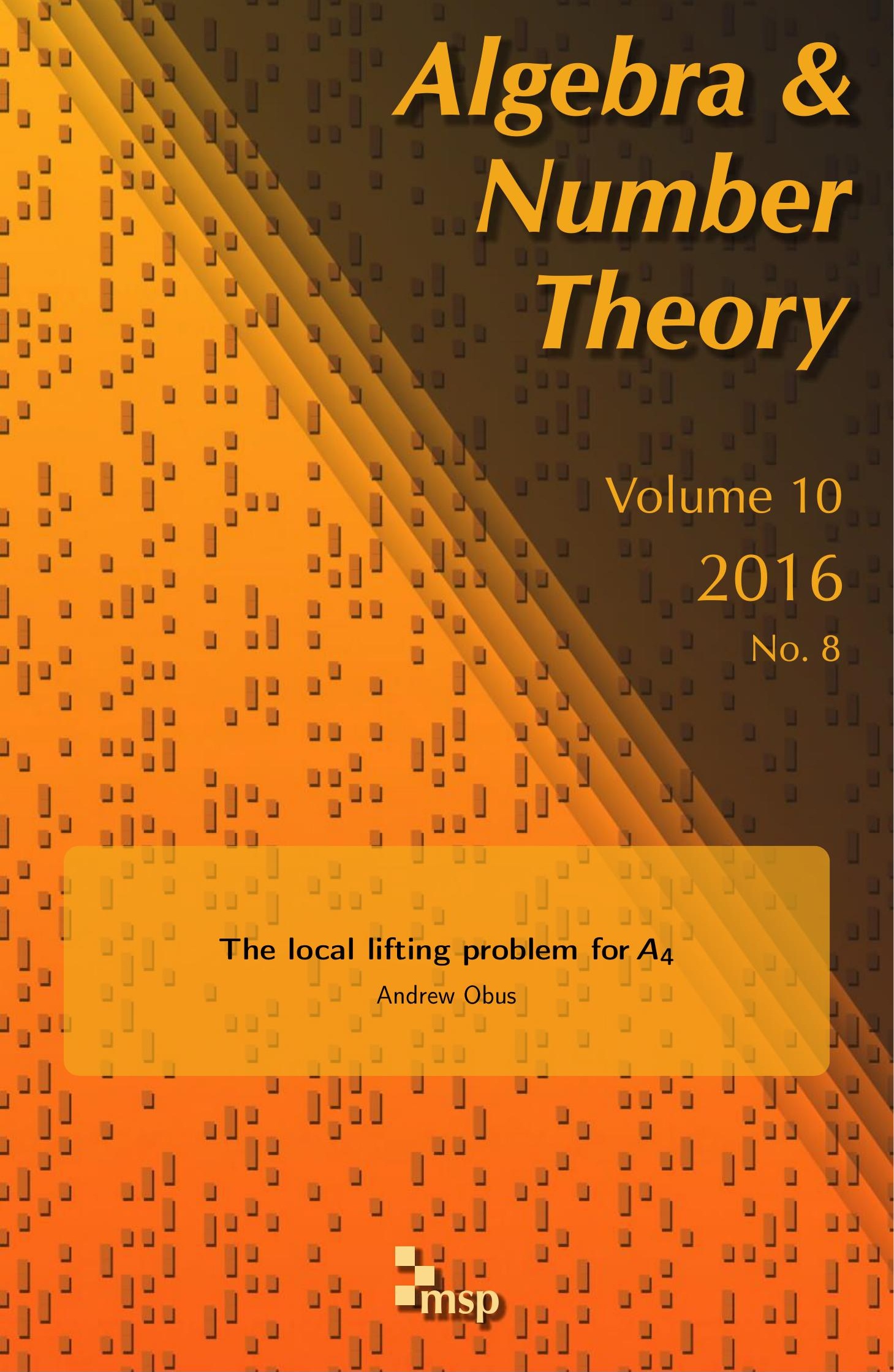




\title{
The local lifting problem for $A_{4}$
}

\author{
Andrew Obus
}

\begin{abstract}
We solve the local lifting problem for the alternating group $A_{4}$, thus showing that it is a local Oort group. Specifically, if $k$ is an algebraically closed field of characteristic 2 , we prove that every $A_{4}$-extension of $k \llbracket s \rrbracket$ lifts to characteristic zero.
\end{abstract}

\section{Introduction}

This paper concerns lifting Galois extensions of power series rings from characteristic $p$ to characteristic zero, the so-called local lifting problem:

Problem 1.1. (The local lifting problem) Let $k$ be an algebraically closed field of characteristic $p$ and $G$ a finite group. Let $k \llbracket z \rrbracket / k \llbracket s \rrbracket$ be a $G$-Galois extension (that is, $G$ acts on $k \llbracket z \rrbracket$ by $k$-automorphisms with fixed ring $k \llbracket s \rrbracket)$. Does this extension lift to characteristic zero? That is, does there exist a DVR $R$ of characteristic zero with residue field $k$ and a $G$-Galois extension $R \llbracket Z \rrbracket / R \llbracket S \rrbracket$, that reduces to $k \llbracket z \rrbracket / k \llbracket s \rrbracket$ ?

We will refer to a $G$-Galois extension $k \llbracket z \rrbracket / k \llbracket s \rrbracket$ as a local $G$-extension. Basic ramification theory shows that any group $G$ that occurs as the Galois group of a local extension is of the form $P \rtimes \mathbb{Z} / m$, with $P$ a $p$-group and $p \nmid m$. Chinburg et al. [2011] ask, given a prime $p$, for which groups $G$ (of the form $P \rtimes \mathbb{Z} / m$ ) is it true that all local $G$-actions (over all algebraically closed fields of characteristic $p$ ) lift to characteristic zero? Such a group is called a local Oort group (for $p$ ). Due to various obstructions (the Bertin obstruction of [Bertin 1998], the KGB obstruction of [Chinburg et al. 2011], and the Hurwitz tree obstruction of [Brewis and Wewers 2009]), the list of possible local Oort groups is quite limited. In particular, due to [Chinburg et al. 2011, Theorem 1.2; Brewis and Wewers 2009], if a group $G$ is a local Oort group for $p$, then $G$ is either cyclic, dihedral of order $2 p^{n}$, or the alternating group $A_{4}(p=2)$. Cyclic groups are known to be local Oort - this is

The author was supported by NSF FRG grant DMS-1265290. He thanks Florian Pop, David Harbater and the referee for useful comments, and Adam Levine for inadvertently suggesting Corollary 1.4 while making a joke about paper sizes.

MSC2010: primary 14H37; secondary 12F10, 13B05, 14B12.

Keywords: local lifting problem, Oort group, Artin-Schreier extension. 
the so-called Oort conjecture, proven by Obus-Wewers [2014] and Pop [2014]. Dihedral groups of order $2 p$ are known to be local Oort for odd $p$ due to Bouw and Wewers [2006] and for $p=2$ due to Pagot [2002]. The group $D_{9}$ is local Oort by [Obus 2015]. Our main theorem is:

Theorem 1.2. If $k$ is an algebraically closed field of characteristic 2, then every $A_{4}$-extension of $k \llbracket s \rrbracket$ lifts to characteristic zero. That is, the group $A_{4}$ is a local Oort group for $p=2$.

This result was announced by Bouw (see the beginning of [Bouw and Wewers 2006]), but the proof has not been written down. Our proof uses a simple idea that avoids the "Hurwitz tree" machinery of [Bouw and Wewers 2006]. Namely, one first classifies local $A_{4}$-extensions by what we call their "break" (this is a jump in the higher ramification filtration). One then uses the following strategy of Pop [2014], sometimes known as the "Mumford method": First, make an equicharacteristic deformation of a local $A_{4}$-extension such that, generically, the break of the extension goes down. If one can lift the local extensions arising from the generic fiber of this deformation, Pop's work shows that one can lift the original extension. On the other hand, we show explicitly that local $A_{4}$-extensions with small break lift. An induction finishes the proof.

We remark that Florian Pop has his own similar proof of Theorem 1.2, which was communicated to the author after the first draft of this paper was written (see Remark 5.3).

The main motivation for the local lifting problem is the following global lifting problem, about deformation of curves with an action of a finite group (or equivalently, deformation of Galois branched covers of curves).

Problem 1.3. (The global lifting problem) Let $X / k$ be a smooth, connected, projective curve over an algebraically closed field of characteristic $p$. Suppose a finite group $\Gamma$ acts on $X$. Does $(X, \Gamma)$ lift to characteristic zero? That is, does there exist a DVR $R$ of characteristic zero with residue field $k$ and a relative projective curve $X_{R} / R$ with $\Gamma$-action, such that $X_{R}$, along with its $\Gamma$-action, reduces to $X$ ?

It is a major result of Grothendieck [SGA 1 1971, XIII, Corollaire 2.12] that the global lifting problem can be solved whenever $\Gamma$ acts with tame (prime-to- $p$ ) inertia groups, and $R$ can be taken to be the Witt ring $W(k)$. More generally, the localglobal principle states that $(X, \Gamma)$ lifts to characteristic zero over $R$, a complete DVR, if and only if the local lifting problem holds (over $R$ ) for each point of $X$ with nontrivial stabilizer in $\Gamma$. Specifically, if $x$ is such a point, then its complete local ring is isomorphic to $k \llbracket z \rrbracket$. The stabilizer $I_{x} \subseteq \Gamma$ acts on $k \llbracket z \rrbracket$ by $k$-automorphisms, and we check the local lifting problem for the local $I_{x}$-extension $k \llbracket z \rrbracket / k \llbracket z \rrbracket^{I_{x}}$. Thus, the global lifting problem is reduced to the local lifting problem. Proofs of 
the local-global principle have been given by Bertin and Mézard [2000], Green and Matignon [1998], and Garuti [1996].

One consequence of the local-global principle and Theorem 1.2 is the following:

Corollary 1.4. The groups $A_{4}$ and $A_{5}$ are so-called Oort groups for every prime. That is, if $\Gamma \in\left\{A_{4}, A_{5}\right\}$ acts on a smooth projective curve $X$ over an algebraically closed field of positive characteristic $p$, then $(X, \Gamma)$ lifts to characteristic zero.

Proof. By the local-global principle (see also [Chinburg et al. 2008, Theorem 2.4]), it suffices to show that every cyclic-by- $p$ subgroup of $A_{4}$ or $A_{5}$ is a local Oort group for $p$. The only subgroups of $A_{4}$ of this form for any $p$ are isomorphic to the trivial group, $\mathbb{Z} / 2, \mathbb{Z} / 2 \times \mathbb{Z} / 2, \mathbb{Z} / 3$, or $A_{4}$. The subgroups of $A_{5}$ of this form are isomorphic to the trivial group, $\mathbb{Z} / 2, \mathbb{Z} / 2 \times \mathbb{Z} / 2, \mathbb{Z} / 3, \mathbb{Z} / 5, D_{3}, A_{4}$, and $D_{5}$. All of these are local Oort groups for the relevant primes, as has been noted above.

Conventions and notation. Throughout, $k$ is an algebraically closed field of characteristic 2. The ring $R$ is a large enough complete discrete valuation ring of characteristic zero with residue field $k$, maximal ideal $\mathfrak{m}$, and uniformizer $\pi$. We normalize the valuation $v$ on $R$ so that $v(2)=1$. In any polynomial or power series ring with coefficients in $R$, the expression $o(x)$ for $x \in R$ means a polynomial or power series with coefficients in $x \mathrm{~m}$.

The ring $k \llbracket t \rrbracket$ is always a $\mathbb{Z} / 3$-extension of $k \llbracket s \rrbracket$ with $t^{3}=s$. Likewise, $R \llbracket T \rrbracket$ is always a $\mathbb{Z} / 3$-extension of $R \llbracket S \rrbracket$ with $T^{3}=S$. If $k \llbracket z \rrbracket / k \llbracket s \rrbracket$ is an extension, it is always assumed to contain $k \llbracket t \rrbracket$. Our convention for variables is that lowercase letters represent the reduction of capital letters from characteristic 0 to characteristic 2 (e.g., $t$ is the reduction of $T$ ).

We write $\zeta_{3}$ for a primitive third root of unity in any ring.

\section{A4-extensions}

We start with the basic structure theory of $A_{4}$-extensions.

\section{A4-extensions in characteristic 2.}

Lemma 2.1. Let $K \subseteq L \subseteq M$ be a tower of field extensions of characteristic 2 such that $L / K$ is $\mathbb{Z} / 3-$ Galois and $M / L$ is $\mathbb{Z} / 2$-Galois. Let $\sigma$ be a generator of $\operatorname{Gal}(L / K)$. For $\ell \in L$, let $\bar{\ell}$ denote the image of $\ell$ in $L /(F-1) L$, where $F$ is Frobenius. Suppose $M \cong L[x] /\left(x^{2}-x-a\right)$, and let $d$ be the dimension of the $\mathbb{F}_{2}$-vector space generated by $\bar{a}, \overline{\sigma(a)}$, and $\overline{\sigma^{2}(a)}$. If $N$ is the Galois closure of $M$ over $L$, then $\operatorname{Gal}(N / K)$ can be expressed as a semidirect product

$$
\operatorname{Gal}(N / K) \cong(\mathbb{Z} / 2)^{d} \rtimes \mathbb{Z} / 3
$$


Proof. By the Schur-Zassenhaus theorem, it suffices to prove $\operatorname{Gal}(N / L) \cong(\mathbb{Z} / 2)^{d}$. But $N / L$ is clearly generated by Artin-Schreier roots of $a, \sigma(a)$, and $\sigma^{2}(a)$. Thus the result follows from Artin-Schreier theory.

Corollary 2.2. If $d=2$ in Lemma 2.1, then $\operatorname{Gal}(N / K) \cong A_{4}$.

Proof. The group $\operatorname{Gal}(N / K)$ must be a semidirect product $(\mathbb{Z} / 2)^{2} \rtimes \mathbb{Z} / 3$ that is nonabelian (as there exists a non-Galois subextension). The only such group is $A_{4}$.

If $K=k((s))$ in Lemma 2.1 above, then after a change of variable, we may assume that $L=k((t))$ with $t^{3}=s$. Then, it is easy to see that an Artin-Schreier representative $a$ of $M / L$ may be chosen uniquely such that $a \in t^{-1} k\left[t^{-1}\right]$ and $a$ has only odd-degree terms. We say that such an $a$ is in standard form. In this case, a standard exercise shows that the break in the higher ramification filtration of $M / L$ (i.e., the largest $i$ such that the higher ramification group $G_{i}$ is nontrivial) occurs at $\operatorname{deg}(a)$, thought of as a polynomial in $t^{-1}$.

Corollary 2.3. Suppose $K=k((s))$ and $L=k((t))$. Suppose $a \in t^{-1} k\left[t^{-1}\right] \subseteq L$ is in standard form. Using the notation of Lemma 2.1, we have $\operatorname{Gal}(N / K) \cong A_{4}$ if and only if a has no nonzero terms of degree divisible by 3.

Proof. Since linear combinations of elements of $L$ in standard form are also in standard form, Lemma 2.1 and Corollary 2.2 imply that $\operatorname{Gal}(N / K)=A_{4}$ if and only if the $\mathbb{F}_{2}$-subspace $V$ of $L$ generated by $a, \sigma(a)$, and $\sigma^{2}(a)$ has dimension 2 . If $a$ has no nonzero terms of degree divisible by 3 , then $a+\sigma(a)+\sigma^{2}(a)=0$ is the only $\mathbb{F}_{2}$-linear relation that holds among the conjugates of $a$, so $\operatorname{dim} V=2$ (note that $a \neq 0$ since it is an Artin-Schreier representative of $M / L$ ). Conversely, if $a$ has a nonzero term of degree divisible by 3 , then either no $\mathbb{F}_{2}$-linear relation holds, or $a \in k\left((s)\right.$ ) (in which case $a=\sigma(a)=\sigma^{2}(a)$ ). In either case, $\operatorname{dim} V \neq 2$.

If $d=2$ in the context of Lemma 2.1, then we say that $a \in L$ gives rise to the $A_{4}$ extension $N / K$. By abuse of notation, if $K \cong k((s))$, we say that the break of $N / K$ is the ramification break of $M / L$. This is the same as the unique ramification break of $N / L$ in either the upper or lower numbering. Furthermore, if $K=k((s))$ and $N=k((z))$, we also say that $a$ gives rise to the extension $k \llbracket z \rrbracket / k \llbracket s \rrbracket$.

Proposition 2.4. If $K=k((s))$ and $N / K$ is an $A_{4}$-extension with break $=v$, then $v \equiv 1$ or $5(\bmod 6)$.

Proof. If $a$ gives rise to $N / K$ and is in standard form, we know that $v$ is the degree of $a$ in $t^{-1}$. This must be odd, and by Corollary 2.3, it cannot be divisible by 3 . 
$A_{4}$-extensions in characteristic zero. The story in characteristic zero (or odd characteristic) is completely analogous. We state the result for reference and omit the proof, which is the same as in Lemma 2.1 with Kummer theory substituted for Artin-Schreier theory.

Proposition 2.5. Let $K \subseteq L \subseteq M$ be a tower of separable field extensions of characteristic $\neq 2$ such that $L / K$ is $\mathbb{Z} / 3$-Galois and $M / L$ is $\mathbb{Z} / 2$-Galois. Let $\sigma$ be a generator of $\operatorname{Gal}(L / K)$. For $\ell \in L^{\times}$, let $\bar{\ell}$ denote the image of $\ell$ in $L^{\times} /\left(L^{\times}\right)^{2}$. Suppose $M \cong L[x] /\left(x^{2}-a\right)$, and let $d$ be the dimension of the $\mathbb{F}_{2}$-subspace of $L^{\times} /\left(L^{\times}\right)^{2}$ generated by $\bar{a}, \overline{\sigma(a)}$, and $\overline{\sigma^{2}(a)}$. If $N$ is the Galois closure of $M$ over $L$, then $\operatorname{Gal}(N / K)$ can be expressed as a semidirect product: $\operatorname{Gal}(N / K) \cong$ $(\mathbb{Z} / 2)^{d} \rtimes \mathbb{Z} / 3$. In particular, if $d=2$, then $\operatorname{Gal}(N / K) \cong A_{4}$.

In the context of Proposition 2.5, we again say that $a \in L$ gives rise to $N / K$.

\section{Characteristic 2 deformations}

For this section, let $K, L, M, N$ be as in Lemma 2.1, with $K=k((s)), L=k((t))$, and $N=k((z))$. Suppose $\operatorname{Gal}(N / K) \cong A_{4}$, and $N / K$ is given rise to by $a \in t^{-1} k\left[t^{-1}\right]$ in standard form. Let $v$ be the break of $N / K$. Our goal is to prove the following proposition.

Proposition 3.1. Suppose that $v>6$ and all $A_{4}$-extensions $N^{\prime} / K$ with break $\leq v-6$ lift to characteristic zero. Then $N / K$ lifts to characteristic zero.

Our proof follows an idea of Pop [2014]. As in [Pop 2014; Obus 2015], we make a deformation in characteristic 2 so that the generic fiber has "milder" ramification than the special fiber.

Proposition 3.2. Let $\mathscr{A}=k \llbracket \varpi, s \rrbracket \supseteq k \llbracket s \rrbracket$, and let $\mathscr{K}=\operatorname{Frac}(\mathscr{A})$. There exists an $A_{4}$-extension $\mathcal{N} / \mathscr{K}$, with $\mathcal{N} \supseteq N$, having the following properties:

(1) The unique $\mathbb{Z} / 3$-subextension $\mathscr{L} / \mathscr{K}$ of $\mathcal{N} / \mathscr{K}$ is given by $\mathscr{L}=\mathscr{K}[t] \subseteq \mathcal{N}$.

(2) If $\mathscr{C}$ is the integral closure of $\mathscr{A}$ in $\mathcal{N}$, we have $\mathscr{C} \cong k \llbracket \varpi, z \rrbracket$. In particular, $(\mathscr{C} /(\varpi)) /(\mathscr{A} /(\varpi))$ is $A_{4}$-isomorphic to the original extension $k \llbracket z \rrbracket / k \llbracket s \rrbracket$.

(3) Let $\mathscr{B}=\mathscr{A}[t] \subseteq \mathscr{L}$, let $\mathscr{R}=\mathscr{A}\left[\varpi^{-1}\right]$, let $\mathscr{Y}=\mathscr{B}\left[\varpi^{-1}\right]$, and let $\mathscr{T}=\mathscr{C}\left[\varpi^{-1}\right]$. Then $\mathscr{T} / \mathscr{R}$ is an $A_{4}$-extension of Dedekind rings, branched at 2 maximal ideals. Above the ideal (s), the inertia group is $A_{4}$, and the break is $v-6$. The other branched ideal has inertia group $\mathbb{Z} / 2 \times \mathbb{Z} / 2$, unique ramification break $=1$, and can be chosen to be of the form $\left(s-\mu^{3}\right)$, where $\mu \in \varpi^{2} k \llbracket \varpi^{2} \rrbracket \backslash\{0\}$ is arbitrary.

Proof. Define $\mathscr{L}$ by adjoining $t$ to $\mathscr{K}$. We proceed by deforming $a$ to an element of $\mathscr{L}$. Let $\mu \in \varpi^{2} k \llbracket \varpi^{2} \rrbracket \backslash\{0\}$. Let $a^{\prime}=a / t^{-6}=a / s^{-2}$, and deform $a$ to the element $\tilde{a}:=a^{\prime}\left(s-\mu^{3}\right)^{-2}=a^{\prime} \prod_{\alpha=1}^{3}\left(\zeta_{3}^{\alpha} t-\mu\right)^{-2} \in \mathscr{B}_{(\varpi)} \subseteq \mathscr{L}$. Note that $\tilde{a}$ reduces to 
$a(\bmod \varpi)$. Observe also that $\operatorname{Gal}(\mathscr{L} / \mathscr{K}) \cong \mathbb{Z} / 3$, and the $\mathbb{F}_{2}$-vector space generated by the images of $a^{\prime}$ (and thus $\tilde{a}$ ) under this Galois action has dimension 2. By Corollary 2.2, $\tilde{a} \in \mathscr{L}$ gives rise to an $A_{4}$-extension $\mathcal{N} / \mathscr{K}$. We claim that this is the extension we seek.

Property (1) is obvious. To show property (3), first note that $\mathscr{Y} / \mathscr{R}$ is branched exactly above the ideal $(s)$. The $\mathbb{Z} / 2$-subextensions of $\mathcal{N} / \mathscr{L}$ are the Artin-Schreier extensions corresponding to $\tilde{a}, \sigma(\tilde{a})$, and $\sigma^{2}(\tilde{a})$, where $\sigma$ generates $\operatorname{Gal}(\mathscr{L} / \mathscr{K})$. Each of these is ramified at most above the ideals $(t)$ and $\left(\zeta_{3}^{\alpha} t-\mu\right)$, for $\alpha \in\{1,2,3\}$. We will see in the next paragraph that all of these ideals ramify in each $\mathbb{Z} / 2$-subextension. Thus the ramification groups of $\mathscr{T} / \mathscr{S}$ above these ideals are all $\mathbb{Z} / 2 \times \mathbb{Z} / 2$. Since the three $\mathbb{Z} / 2$-subextensions are Galois conjugate over $\mathscr{K}$, there can only be one higher ramification jump for each ideal, and it is determined, say, by the Artin-Schreier subextension corresponding to $\tilde{a}$.

To determine the ramification, we consider the Artin-Schreier extension of the complete discrete valuation field $k((\varpi))((t))\left(\right.$ resp. $k((\varpi))\left(\left(\zeta_{3}^{\alpha} t-\mu\right)\right)$ for $\alpha \in$ $\{1,2,3\})$ given by $\tilde{a}$. Since $t$ is a unit in $k((\varpi)) \llbracket \zeta_{3}^{\alpha} t-\mu \rrbracket$ for any $\alpha$ and $\zeta_{3}^{\alpha} t-\mu$ is a unit in $k((\varpi)) \llbracket t \rrbracket$ and in $k((\varpi)) \llbracket \zeta_{3}^{\alpha^{\prime}} t-\mu \rrbracket$ for any $\alpha^{\prime} \neq \alpha$ in $\{1,2,3\}$, the degree of the pole of $\tilde{a}$ with respect to $t$ (resp. $\zeta_{3}^{\alpha} t-\mu$ ) is $v-6$ (resp. 2). Since $v-6$ is odd, we have that the Artin-Schreier extension of $k((\varpi))((t))$ given by $\tilde{a}$ ramifies and has ramification break $=v-6$. To calculate the ramification break for the corresponding extension of $k((\varpi))\left(\left(\zeta_{3}^{\alpha} t-\mu\right)\right)$, we assume $\alpha=3$ for simplicity and we write $\tilde{a}$ as a Laurent series in $(t-\mu)$. Note that $\tilde{a}=t^{-1}(t-\mu)^{-2} x^{2}$ for some $x \in k((\varpi)) \llbracket t-\mu \rrbracket^{\times}$, and that

$$
t^{-1}=\mu^{-1}+\mu^{-2}(t-\mu)+\text { higher order terms in }(t-\mu) .
$$

So

$$
\tilde{a}=c \mu^{-1}(t-\mu)^{-2}+c \mu^{-2}(t-\mu)^{-1}+\theta,
$$

where $\theta \in k((\varpi)) \llbracket t-\mu \rrbracket$ and $c \in k((\varpi))$ is the "constant" term of $x^{2}$ (in fact, it is easy to see that $\left.c \in k\left(\left(\mu^{2}\right)\right)=k\left(\left(\varpi^{4}\right)\right)\right)$. Let $b=\sqrt{c \mu^{-1}}(t-\mu)^{-1}$. After replacing $\tilde{a}$ with $\tilde{a}+b^{2}-b$, which does not change the Artin-Schreier extension, we see that $\tilde{a}$ has a simple pole (since $c \neq \mu^{3}$, the principal part does not vanish). So this extension ramifies with ramification break $=1$. This shows property (3).

For property (2), it suffices by [Green and Matignon 1998, I, Theorem 3.4] to show that the total degree of the different of $\mathscr{T} / \mathscr{R}$ is equal to the degree of the different of $N / K$. Clearly, we may replace $\mathscr{R}$ by $\mathscr{S}$ and $K$ by $L$. Call these degrees $\delta_{\mathcal{T} / \mathscr{S}}$ and $\delta_{N / L}$, respectively.

Since the ramification break of $M / L$ is $v$, and $N / L$ is the compositum of Galois conjugates of $M / L$, we have that $N / L$ has $v$ as its single ramification break in the upper numbering, and all nontrivial higher ramification groups of $N / L$ have 
order 4. Using Serre's different formula [Serre 1968, IV, Proposition 4], we obtain $\delta_{N / L}=3(v+1)$.

For $\delta_{\mathcal{T} / \varphi}$, we add up the contributions from the different branched ideals separately. For the ideal $(t)$, arguing as in the previous paragraph, we have a $\mathbb{Z} / 2 \times \mathbb{Z} / 2$ extension with single ramification break $=v-6$. This gives a contribution of $3(v-5)$ to $\delta_{\mathscr{T} / \varphi}$. For each of the branched ideals $\left(\zeta^{\alpha} t-\mu\right)(\alpha \in\{1,2,3\})$, we have ramification group $\mathbb{Z} / 2 \times \mathbb{Z} / 2$ with ramification break $=1$. Using Serre's different formula again, we get a contribution of $3 \cdot 3 \cdot 2=18$ to $\delta_{\mathscr{T} / \varphi}$. Thus $\delta_{\mathscr{T} / \varphi}=3(v-5)+18=\delta_{N / L}$, and we are done.

We omit the proof of the next proposition, which follows from Proposition 3.2 exactly as Theorem 3.6 follows from Key Lemma 3.2 in [Pop 2014].

Proposition 3.3. Let $Y \rightarrow W$ be a branched $A_{4}$-cover of projective smooth $k$ curves. Suppose that the local inertia at each totally ramified point is an extension $k \llbracket z \rrbracket / k \llbracket s \rrbracket$ having break $\leq v$ and given rise to by an Artin-Schreier generator in standard form divisible by $t^{-6}$ in $k\left[t^{-1}\right]$. Set $\mathscr{W}=W \times_{k} k \llbracket \varpi \rrbracket$. Then there is an $A_{4}$-cover of projective smooth $k \llbracket \varpi \rrbracket$-curves $\mathrm{Y} \rightarrow \mathbb{W}$ with special fiber $Y \rightarrow W$ such that the totally ramified points on the generic fiber $\mathscr{Y}_{\eta} \rightarrow W_{\eta}$ have break $\leq v-6$.

Before we prove Proposition 3.1, we recall Harbater-Katz-Gabber covers (or HKG-covers) from [Katz 1986]. Let $G \cong P \rtimes \mathbb{Z} / m$, with $P$ a $p$-group and $p \nmid m$. If $k \llbracket z \rrbracket / k \llbracket s \rrbracket$ is a local $G$-extension, then the associated HKG-cover is the unique branched $G$-cover $X \rightarrow \mathbb{P}_{k}^{1}$ tamely ramified of index $m$ above $s=\infty$ and totally ramified above $s=0$ (where $s$ is a coordinate on $\mathbb{P}_{k}^{1}$ ), such that the formal completion of $X \rightarrow \mathbb{P}_{k}^{1}$ above 0 yields $k \llbracket z \rrbracket / k \llbracket s \rrbracket$.

Proof of Proposition 3.1. The proof is essentially the same as the proof of [Obus 2015, Proposition 1.11], which itself is adapted from [Pop 2014]. We include it here for completeness.

Let $Y \rightarrow W=\mathbb{P}^{1}$ be the Harbater-Katz-Gabber cover associated to $k \llbracket z \rrbracket / k \llbracket s \rrbracket$, let $\mathscr{Y} \rightarrow \mathscr{W}$ be the $A_{4}$-cover over $k \llbracket \varpi \rrbracket$ guaranteed by Proposition 3.3, let $\overline{\mathscr{Y}} \rightarrow \bar{W}$ be its base change to the integral closure of $k \llbracket \varpi \rrbracket$ in $\overline{k((\varpi))}$, and let $\overline{\mathscr{Y}_{\eta}} \rightarrow \overline{\mathscr{W}_{\eta}}$ be the generic fiber of $\mathscr{Y} \rightarrow \mathscr{W}$. Recall that we assume that every local $A_{4}$-extension with break $\leq v-6$ lifts to characteristic zero. Furthermore, by [Pagot 2002] and the theory of tame ramification, every abelian extension of $k \llbracket s \rrbracket$ (and thus of $\overline{k((\varpi))}((s)))$ with Galois group a proper subgroup of $A_{4}$ lifts to characteristic zero. So the local-global principle tells us that $\overline{\mathscr{Y}_{\eta}} \rightarrow \overline{\mathcal{W}_{\eta}}$ lifts to a cover $\mathscr{Y}_{\mathscr{O}_{1}} \rightarrow \mathcal{W}_{\mathscr{O}_{1}}$ over some characteristic zero complete discrete valuation ring $\mathrm{O}_{1}$ with residue field $\overline{k((\varpi))}$. Then, [Pop 2014, Lemma 4.3] shows that we can "glue" the covers $\bar{y} \rightarrow \bar{W}$ and $\mathscr{Y}_{\mathscr{O}_{1}} \rightarrow \mathcal{W}_{\mathscr{O}_{1}}$ along the generic fiber of the former and the special fiber of the latter, in order to get a cover defined over a rank two characteristic zero valuation ring 0 with residue field $k$ lifting $Y \rightarrow W$ (compare [Pop 2014, p. 319, second 
paragraph $]$ ). Note that this process works starting with any $A_{4}$-extension of $k \llbracket s \rrbracket$ with break $=v$, and that such extensions can be parametrized by some affine space $\mathbb{A}^{N}$ (with one coordinate corresponding to each possible coefficient in an entry of an Artin-Schreier generator in standard form).

To conclude, we remark that [Pop 2014, Proposition 4.7] and its setup carry through exactly in our situation, with our $\mathbb{A}^{N}$ playing the role of $\mathbb{A}^{|l|}$ in [Pop 2014]. Indeed, we have that the analog of $\Sigma_{\iota}$ in that proposition contains all closed points, by the paragraph above. Thus we can in fact lift $Y \rightarrow W$ over a discrete characteristic zero valuation ring. Applying the easy direction of the local-global principle, we obtain a lift of $k \llbracket z \rrbracket / k \llbracket s \rrbracket$. This concludes the proof of Proposition 3.1.

\section{The form of a lift}

We start by reviewing lifts of $\mathbb{Z} / 2$-extensions of $k \llbracket t \rrbracket$. The following lemma is well-known, but difficult to cite directly from the literature. We provide a proof.

Lemma 4.1. Let $k((u)) / k((t))$ be a $\mathbb{Z} / 2$-extension with Artin-Schreier generator $a \in t^{-1} k\left[t^{-1}\right]$ in standard form and ramification break $=v$. Let $A$ be a lift of a to $T^{-1} R\left[T^{-1}\right]$ of degree $v$. If $\Phi \in 1+T^{-1} \mathfrak{m}\left[T^{-1}\right]$ has degree $v$ or $v+1$ and there exists $H \in 1+T^{-1} \mathfrak{m}\left[T^{-1}\right]$ such that

$$
\Phi=H^{2}+4 A+o(4),
$$

then the normalization of $R \llbracket T \rrbracket$ in $M:=\operatorname{Frac}(R \llbracket T \rrbracket)[\sqrt{\Phi}]$ is a lift of $k \llbracket u \rrbracket / k \llbracket t \rrbracket$ to characteristic zero. Furthermore, $\Phi$ has simple roots.

Proof. The extension $k((u)) / k((t))$ is given by adjoining an element $y$ such that $y^{2}-y=a$. Making a substitution $\sqrt{\Phi}=H+2 Y$, the expression for $\Phi$ given in the lemma yields

$$
H^{2}+4 H Y+4 Y^{2}=H^{2}+4 A+o(4),
$$

or $Y^{2}-Y=A+o(1)$. Thus we see that the normalization of $R \llbracket T \rrbracket_{(\pi)}$ in $M$ gives $k((u)) / k((t))$ upon reduction modulo $\pi$. By Serre's different formula [Serre 1968, IV, Proposition 4], the degree of the different of $k \llbracket u \rrbracket / k \llbracket t \rrbracket$ is $v+1$. On the other hand, the normalization of $R \llbracket T \rrbracket \otimes_{R} \operatorname{Frac}(R)$ in $M$ is branched at at most $v+1$ maximal ideals, corresponding to the roots of $\Phi$ and also 0 if $\Phi$ has degree $v$. Since this is a tamely ramified $\mathbb{Z} / 2$-extension, the degree of its different is at most $v+1$. By [Green and Matignon 1998, I, 3.4], the degree of the different is exactly $v+1$ and the normalization of $R \llbracket T \rrbracket$ in $M$ is a lift of $k \llbracket u \rrbracket / k \llbracket t \rrbracket$. This also shows that the roots of $\Phi$ are all simple.

For Proposition 4.2 below, recall that $s=t^{3}$ and $S=T^{3}$. 
Proposition 4.2. Let $k \llbracket z \rrbracket / k \llbracket s \rrbracket$ be a local $A_{4}$-extension with break $=v$ given rise to by $a \in t^{-1} k\left[t^{-1}\right]$ in standard form. If $F\left(T^{-1}\right)$ and $H\left(T^{-1}\right)$ are in $1+T^{-1} \mathfrak{m}\left[T^{-1}\right]$ such that $F$ has degree $(v+1) / 2$ and

$$
F\left(\zeta_{3} T^{-1}\right) F\left(\zeta_{3}^{2} T^{-1}\right)=H^{2}+4 A+o(4),
$$

where $A$ is a lift of a to $T^{-1} R\left[T^{-1}\right]$ of degree $v$, then the normalization of $R \llbracket S \rrbracket$ in the $A_{4}$-extension of $\operatorname{Frac}(R \llbracket S \rrbracket)$ given rise to by $F\left(\zeta_{3} T^{-1}\right) F\left(\zeta_{3}^{2} T^{-1}\right)$ is a lift of $k \llbracket z \rrbracket / k \llbracket s \rrbracket$ to characteristic zero.

Proof. Let the local $\mathbb{Z} / 2$-extension $k \llbracket u \rrbracket / k \llbracket t \rrbracket$ be given by normalizing $k \llbracket t \rrbracket$ in the Artin-Schreier $\mathbb{Z} / 2$-extension of $k((t))$ given by $a$. Let $L=\operatorname{Frac}(R \llbracket T \rrbracket)$. By Lemma 4.1, normalizing $R \llbracket T \rrbracket$ in the degree 2 Kummer extension $M / L$ given by some polynomial $\Phi \in 1+T^{-1} \mathfrak{m}\left[T^{-1}\right]$ of degree $v+1$ in $T^{-1}$ such that $\Phi=$ $H^{2}+4 A+o(4)$ with $A$ as in the proposition gives a lift of $k \llbracket u \rrbracket / k \llbracket t \rrbracket$ to characteristic zero, and such a $\Phi$ has simple roots.

Let $\sigma$ generate $\operatorname{Gal}(L / \operatorname{Frac}(R \llbracket S \rrbracket))$ and, by abuse of notation, $\operatorname{Gal}(k((t)) / k((s)))$. Write $\Phi=F\left(\zeta_{3} T^{-1}\right) F\left(\zeta_{3}^{2} T^{-1}\right)$ for some polynomial $F \in 1+T^{-1} \mathfrak{m}\left[T^{-1}\right]$ of degree $(v+1) / 2$ as in the proposition. Then $\Phi$ has simple roots, and thus $F\left(T^{-1}\right)$, $F\left(\zeta_{3} T^{-1}\right)$, and $F\left(\zeta_{3}^{2} T^{-1}\right)$ have pairwise disjoint simple roots. Consequently, the $\mathbb{F}_{2}$-subspace of $L^{\times} /\left(L^{\times}\right)^{2}$ generated by $\Phi, \sigma(\Phi)$, and $\sigma^{2}(\Phi)$ has dimension 2. By Proposition 2.5, this is equivalent to the Galois closure $N$ (over Frac $(R \llbracket S \rrbracket)$ ) of $M$ having Galois group $A_{4}$.

Let $k\left(\left(u^{\prime}\right)\right) / k((t))$ be the Artin-Schreier extension given by $\sigma(a)$. Clearly, the normalization of $R \llbracket T \rrbracket$ in $\operatorname{Frac}(R \llbracket T \rrbracket)[\sqrt{\sigma(\Phi)}]$ is a lift of $k \llbracket u^{\prime} \rrbracket / k \llbracket t \rrbracket$. Note that $k \llbracket z \rrbracket$ is the normalization of $k \llbracket t \rrbracket$ in the compositum of $k((u))$ and $k\left(\left(u^{\prime}\right)\right)$. Analogously, $N:=\operatorname{Frac}(R \llbracket T \rrbracket)(\sqrt{\Phi}, \sqrt{\sigma(\Phi)})$ is the $A_{4}$-extension given rise to by $\Phi$. Now, $\Phi$ and $\sigma(\Phi)$ have exactly $(v+1) / 2$ zeroes in common. Thus [Green and Matignon 1998, I, Theorem 5.1] shows that the normalization of $R \llbracket T \rrbracket$ in $N$ is a lift of the Klein four extension $k \llbracket z \rrbracket / k \llbracket t \rrbracket$ (and is isomorphic to $R \llbracket Z \rrbracket / R \llbracket T \rrbracket$ for $Z$ reducing to $z$ ). We conclude that $R \llbracket Z \rrbracket / R \llbracket S \rrbracket$ is a lift of $k \llbracket z \rrbracket / k \llbracket s \rrbracket$.

\section{Proof of Theorem 1.2}

In this section, let $k \llbracket z \rrbracket / k \llbracket s \rrbracket$ be a local $A_{4}$-extension given rise to by $a \in t^{-1} k\left[t^{-1}\right]$ in standard form. Recall that $\operatorname{deg}(a)=v$, where $v$ is the break in $k \llbracket z \rrbracket / k \llbracket s \rrbracket$. We will prove that $k \llbracket z \rrbracket / k \llbracket s \rrbracket$ lifts to characteristic zero by strong induction on $v$.

Proposition 5.1. If $v=1$, then $k \llbracket z \rrbracket / k \llbracket s \rrbracket$ lifts to characteristic zero.

Proof. Since $v=1$, we have $a=\bar{c}_{1} t^{-1}$, with $\bar{c}_{1} \in k$. By Proposition 4.2, it suffices to find $F\left(T^{-1}\right)$ and $H\left(T^{-1}\right)$ in $1+T^{-1} R\left[T^{-1}\right]$ such that $F$ has degree 1 and

$$
F\left(\zeta_{3} T^{-1}\right) F\left(\zeta_{3}^{2} T^{-1}\right)=H^{2}+4 c_{1} T^{-1}+o(4),
$$


where $c_{1}$ is a lift of $\bar{c}_{1}$ to $R$. This is done by taking $H=1$ and $F=1-4 c_{1} T^{-1}$.

Proposition 5.2. If $v=5$, then $k \llbracket z \rrbracket / k \llbracket s \rrbracket$ lifts to characteristic zero.

Proof. Since $v=5$, we have $a=\bar{c}_{1} t^{-1}+\bar{c}_{5} t^{-5}$, with $\bar{c}_{1}, \bar{c}_{5} \in k$. By Proposition 4.2, it suffices to find $F\left(T^{-1}\right)$ and $H\left(T^{-1}\right)$ in $1+T^{-1} R\left[T^{-1}\right]$ such that $F$ has degree 3 and

$$
F\left(\zeta_{3} T^{-1}\right) F\left(\zeta_{3}^{2} T^{-1}\right)=H^{2}+4 c_{1} T^{-1}+4 c_{5} T^{-5}+o(4),
$$

where each $c_{i}$ is a lift of $\bar{c}_{i}$ to $R$.

Let $b \in R$ be any element such that $v(b)=\frac{2}{5}$. Write

$$
F\left(T^{-1}\right)=1+a_{1} T^{-1}+a_{2} T^{-2}+a_{3} T^{-3},
$$

where

$$
a_{1}=-2 b-4 c_{1}, \quad a_{2}=b^{2}, \quad a_{3}=-4 c_{5} / b^{2} .
$$

Note that $v\left(a_{1}\right)=\frac{7}{5}, v\left(a_{2}\right)=\frac{4}{5}$, and $v\left(a_{3}\right)=\frac{6}{5}$. Then

$$
\begin{aligned}
F\left(\zeta_{3} T^{-1}\right) F\left(\zeta_{3}^{2} T^{-1}\right) & =1-a_{1} T^{-1}-a_{2} T^{-2}+a_{2}^{2} T^{-4}-a_{2} a_{3} T^{-5}+o(4) \\
& =1+\left(4 c_{1}+2 b\right) T^{-1}-b^{2} T^{-2}+b^{4} T^{-4}+4 c_{5} T^{-5}+o(4) \\
& =\left(1+b T^{-1}+b^{2} T^{-2}\right)^{2}+4 c_{1} T^{-1}+4 c_{5} T^{-5}+o(4) .
\end{aligned}
$$

We conclude by taking $H=1+b T^{-1}+b^{2} T^{-2}$.

Proof of Theorem 1.2. We use strong induction on the break $=v$ of $k \llbracket z \rrbracket / k \llbracket s \rrbracket$, which only takes values congruent to 1 or 5 modulo 6 (Proposition 2.4). The base cases $v=1$ and $v=5$ are Propositions 5.1 and 5.2, respectively. The induction step is Proposition 3.1.

Remark 5.3. Florian Pop has informed the author of his own proof, which uses much the same method. In place of the deformation in Proposition 3.2, he uses one for which it is slightly more difficult to verify that it yields an $A_{4}$-extension, but which immediately reduces Theorem 1.2 to the case $v=1$ (eliminating the need for Proposition 5.2).

Question 5.4. Given $k$, does there exist a particular DVR $R$ in characteristic zero such that all local $A_{4}$-extensions over $k$ lift over $R$ ? This is known for local $G$ extensions in characteristic $p$ where $G$ is cyclic with $v_{p}(|G|) \leq 2$ (see [Green and Matignon 1998], where it is shown that $W(k)\left[\zeta_{p^{2}}\right]$ works). Since our proof is rather inexplicit, this question remains open for $A_{4}$.

\section{References}

[Bertin 1998] J. Bertin, "Obstructions locales au relèvement de revêtements galoisiens de courbes lisses”, C. R. Acad. Sci. Paris Sér. I Math. 326:1 (1998), 55-58. MR 1649485 
[Bertin and Mézard 2000] J. Bertin and A. Mézard, "Déformations formelles des revêtements sauvagement ramifiés de courbes algébriques”, Invent. Math. 141:1 (2000), 195-238. MR 1767273

[Bouw and Wewers 2006] I. I. Bouw and S. Wewers, "The local lifting problem for dihedral groups", Duke Math. J. 134:3 (2006), 421-452. MR 2254623

[Brewis and Wewers 2009] L. H. Brewis and S. Wewers, "Artin characters, Hurwitz trees and the lifting problem”, Math. Ann. 345:3 (2009), 711-730. MR 2534115

[Chinburg et al. 2008] T. Chinburg, R. Guralnick, and D. Harbater, "Oort groups and lifting problems", Compos. Math. 144:4 (2008), 849-866. MR 2441248

[Chinburg et al. 2011] T. Chinburg, R. Guralnick, and D. Harbater, "The local lifting problem for actions of finite groups on curves”, Ann. Sci. Éc. Norm. Supér. (4) 44:4 (2011), 537-605. MR 2919977

[Garuti 1996] M. A. Garuti, "Prolongement de revêtements galoisiens en géométrie rigide", Compositio Math. 104:3 (1996), 305-331. MR 1424559

[Green and Matignon 1998] B. Green and M. Matignon, "Liftings of Galois covers of smooth curves", Compositio Math. 113:3 (1998), 237-272. MR 1645000

[Katz 1986] N. M. Katz, "Local-to-global extensions of representations of fundamental groups", Ann. Inst. Fourier (Grenoble) 36:4 (1986), 69-106. MR 867916

[Obus 2015] A. Obus, "A generalization of the Oort Conjecture”, Preprint, 2015. arXiv 1502.07623

[Obus and Wewers 2014] A. Obus and S. Wewers, "Cyclic extensions and the local lifting problem", Ann. of Math. (2) 180:1 (2014), 233-284. MR 3194815

[Pagot 2002] G. Pagot, Relèvement en caractéristique zéro d'actions de groupes abéliens de type $(p, \ldots, p)$, Ph.D. thesis, Université Bordeaux I, 2002, Available at http://www.math.u-bordeaux1.fr/ $\sim$ mmatigno/Pagot-These.pdf.

[Pop 2014] F. Pop, “The Oort conjecture on lifting covers of curves", Ann. of Math. (2) 180:1 (2014), 285-322. MR 3194816

[Serre 1968] J.-P. Serre, Corps locaux, Hermann, Paris, 1968. MR 0354618

[SGA 1 1971] A. Grothendieck, Revêtements étales et groupe fondamental (Séminaire de Géométrie Algébrique du Bois Marie 1960-1961), Lecture Notes in Math. 224, Springer, Berlin, 1971. MR 50 \#7129 Zbl 0234.14002

Communicated by Kiran S. Kedlaya

Received 2016-02-17 Revised 2016-06-27 Accepted 2016-07-31

andrewobus@gmail.com Department of Mathematics, University of Virginia, 141 Cabell Drive, Charlottesville, VA 22904, United States 


\section{Algebra \& Number Theory}

msp.org/ant

\section{EDITORS}

MANAGING EDITOR

Bjorn Poonen

Massachusetts Institute of Technology

Cambridge, USA

\author{
EDITORIAL BOARD CHAIR \\ David Eisenbud \\ University of California \\ Berkeley, USA
}

BOARD OF EDITORS

$\begin{aligned} \text { Dave Benson } & \text { University of Aberdeen, Scotland } & \text { Susan Montgomery } & \text { University of Southern California, USA } \\ \text { Richard E. Borcherds } & \text { University of California, Berkeley, USA } & \text { Shigefumi Mori } & \text { RIMS, Kyoto University, Japan } \\ \text { John H. Coates } & \text { University of Cambridge, UK } & \text { Raman Parimala } & \text { Emory University, USA } \\ \text { J-L. Colliot-Thélène } & \text { CNRS, Université Paris-Sud, France } & \text { Jonathan Pila } & \text { University of Oxford, UK } \\ \text { Brian D. Conrad } & \text { Stanford University, USA } & \text { Anand Pillay } & \text { University of Notre Dame, USA } \\ \text { Hélène Esnault } & \text { Freie Universität Berlin, Germany } & \text { Victor Reiner } & \text { University of Minnesota, USA } \\ \text { Hubert Flenner } & \text { Ruhr-Universität, Germany } & \text { Peter Sarnak } & \text { Princeton University, USA } \\ \text { Sergey Fomin } & \text { University of Michigan, USA } & \text { Joseph H. Silverman } & \text { Brown University, USA } \\ \text { Edward Frenkel } & \text { University of California, Berkeley, USA } & \text { Michael Singer } & \text { North Carolina State University, USA } \\ \text { Andrew Granville } & \text { Université de Montréal, Canada } & \text { Vasudevan Srinivas } & \text { Tata Inst. of Fund. Research, India } \\ \text { Joseph Gubeladze } & \text { San Francisco State University, USA } & \text { J. Toby Stafford } & \text { University of Michigan, USA } \\ \text { Roger Heath-Brown } & \text { Oxford University, UK } & \text { Ravi Vakil } & \text { Stanford University, USA } \\ \text { Craig Huneke } & \text { University of Virginia, USA } & \text { Michel van den Bergh } & \text { Hasselt University, Belgium } \\ \text { Kiran S. Kedlaya } & \text { Univ. of California, San Diego, USA } & \text { Marie-France Vignéras } & \text { Université Paris VII, France } \\ \text { János Kollár } & \text { Princeton University, USA } & \text { Kei-Ichi Watanabe } & \text { Nihon University, Japan } \\ \text { Yuri Manin } & \text { Northwestern University, USA } & \text { Efim Zelmanov } & \text { University of California, San Diego, USA } \\ \text { Philippe Michel } & \text { École Polytechnique Fédérale de Lausanne } & \text { Shou-Wu Zhang } & \text { Princeton University, USA }\end{aligned}$

PRODUCTION

production@msp.org

Silvio Levy, Scientific Editor

See inside back cover or msp.org/ant for submission instructions.

The subscription price for 2016 is US $\$ 290$ /year for the electronic version, and $\$ 485 /$ year (+\$55, if shipping outside the US) for print and electronic. Subscriptions, requests for back issues and changes of subscribers address should be sent to MSP.

Algebra \& Number Theory (ISSN 1944-7833 electronic, 1937-0652 printed) at Mathematical Sciences Publishers, 798 Evans Hall \#3840, c/o University of California, Berkeley, CA 94720-3840 is published continuously online. Periodical rate postage paid at Berkeley, CA 94704, and additional mailing offices.

ANT peer review and production are managed by EditFLow ${ }^{\circledR}$ from MSP.

\section{PUBLISHED BY}

- mathematical sciences publishers

nonprofit scientific publishing

http://msp.org/

() 2016 Mathematical Sciences Publishers 


\section{Algebra \& Number Theory}

Volume $10 \quad$ No. $8 \quad 2016$

Tropical independence, II: The maximal rank conjecture for quadrics

DAVID JENSEN and SAM PAYNE

Algebraicity of normal analytic compactifications of $\mathbb{C}^{2}$ with one irreducible curve at infinity

PINAKI MONDAL

The local lifting problem for $A_{4}$

ANDREW OBUS

Syntomic cohomology and $p$-adic regulators for varieties over $p$-adic fields

JAN NEKOVÁŘ and WIESŁAWA NIZIOŁ

Appendix: LAURENT BERGER and FRÉDÉRIC DÉGLISE

Steinberg groups as amalgams

DANIEL ALLCOCK 